\title{
Tecnura
}

\section{Influencia del biodiésel de aceite de fritura usado, sobre las emisiones: índice de opacidad}

\section{Influence of biodiesel made from waste cooking oill on emissions: opacity index}

\author{
Eddy Roberto Cuty Clemente' ${ }^{1}$, Antonio David Mejía Regalado²
}

Fecha de recepción: 25 de julio de 2018

Fecha de aceptación: 12 de noviembre de 2018

Cómo citar: Cuty C., E.R. y Mejía R., A.D. (2019). Influencia del biodiésel de aceite de fritura usado sobre las emisiones: índice de opacidad. Tecnura, 23(59), 60-67. DOI: https://doi.org/10.14483/22487638.13722

\section{Resumen}

Contexto: Entre las fuentes de biodiésel, el aceite de fritura usado es un potencial combustible alternativo para reducir las emisiones de efecto invernadero de los motores diésel sin modificaciones tecnológicas considerables.

Método: Los experimentos se realizaron en condiciones estables, en un motor diésel de un cilindro de cuatro tiempos, mediante tres mezclas de combustible diésel puro con biodiésel obtenido de aceite de fritura usado (B10, B20 y B30) y combustible diésel comercial B5. El motor funcionó a velocidades variables entre 1000-1600 r.p.m., a plena carga y cargas variables del motor correspondientes a la potencia efectiva a 1600 r.p.m.

Resultados: Los resultados experimentales muestran que el índice de opacidad de B10, B20 y B30 a 1600 r.p.m., con velocidad nominal 1600 r.p.m. del motor a carga constante fue menor comparado con el combustible comercial B5 en 0,3\%, $24 \%$ y $46 \%$, respectivamente. Sin embargo, al incrementar la carga del motor a velocidad constante de 1600 rpm, el índice de opacidad alcanzó su máximo valor para B20 comparado con los otros combustibles utilizados.

Conclusiones: Es viable el uso de biodiésel de aceite de fritura usado en mezclas con diésel puro y comercial para disminuir las emisiones tóxicas, como el material particulado (hollín) de los motores diésel en el medio ambiente. Por tanto, la reutilización de dicho aceite disminuye significativamente los problemas medioambientales.

Palabras clave: biodiésel, emisiones, índice de opacidad.

\section{Abstract}

Context: Among the sources of biodiesel, waste cooking oil is a potential alternative fuel to reduce the emission of greenhouse gases from diesel engines without considerable technological changes.

Method: In this study, the experiments were performed under stable conditions on a single-cylinder four-stroke diesel engine using three different blends of pure diesel fuel with biodiesel made from waste cooking oil (B10, B20 and B30) and commercial diesel fuel B5. The engine ran at variable speed between 1000-1600 rpm, at full load and variable engine loads corresponding to the effective power at 1600 rpm.

Results: The experimental results show that smoke opacity of B10, B20, and B30 is lower compared to the commercial B5 at $0.3 \%, 24 \%$ and $46 \%$ respectively, when evaluated at nominal speed $1600 \mathrm{rpm}$

1 Ingeniero mecánico, especialista en Combustibles Alternativos, magíster en Ingeniería Mecánica, doctor en Ingeniería. Docente de la Universidad Nacional de Ingeniería. Lima, Perú. Contacto: ecutyc@uni.edu.pe. ORCID: https://orcid.org/0000-0002-9214-9659

2 Ingeniero mecánico de automotores, maestro en Ciencias con mención en Ingeniería Mecánica-Motores de Combustión Interna. Docente de la Universidad Tecnológica del Perú. Lima, Perú. Contacto: amejiar@utp.edu.pe. ORCID: https://orcid.org/0000-0002-6734-9229 
and at full load. However, by increasing the engine load at a constant speed of 1600 rpm, smoke opacity reached its maximum value with B20 compared to other fuels used.

Conclusions: It is feasible to use biodiesel made with waste cooking oil in blends with pure and commercial diesel to reduce the effects of toxic emissions such as particulate matter (soot) of diesel engines on the environment. Therefore, recycling waste cooking oil significantly reduces environmental problems.

Keywords: biodiesel, emissions, opacity index

\section{INTRODUCCIÓN}

El aumento de la demanda de energía y el deterioro del medio ambiente son factores que conducen a la búsqueda de combustibles alternativos, que reemplacen total o parcialmente los combustibles fósiles convencionales en los motores de combustión interna. Entre las alternativas, el biodiésel es uno de los más investigados debido a que es un producto renovable con elevado potencial de reducción de emisión de gases de efecto invernadero (Arango et al., 2014) y la inhibición de la formación del hollín (Man et al., 2016).

El biodiésel es producido a partir de desechos de aceites vegetales (Núñez, 2012) y animales. La elevada viscosidad de los desechos de aceites utilizados en la producción de biodiesel, puede causar problemas en el bombeo del suministro de combustible, la atomización del combustible y la combustión incompleta cuando la mezcla con el aire no se logra plenamente. Además, los países de la Unión Europea han incrementado sucesivamente el porcentaje de biodiesel en las mezclas, desde 2005 (2\%), en el año $2010(5,57 \%)$ y se proyecta hasta $2020(20 \%)$, como parte de la política de cuidado con el medio ambiente (Behcet et al., 2014).

Existen diferentes métodos para la producción de biodiésel, pero el más usado es el de transesterificación de los aceites usados, cuya finalidad es separar la glicerina del aceite, reducir el punto ebullición, el punto de inflamación y disminuir la viscosidad del aceite usado (Behcet et al., 2014; Nabanita et al., 2014; Gopal et al., 2014; Adaileh y AIQdah, 2012). En la figura 1 se muestra la ecuación general del proceso de transesterificación del aceite usado. En un estudio realizado por Canesin et al. (2014), los resultados sugieren que es posible la obtención de biodiésel de aceites residuales en correspondencia con las normas de la National Association of Petroleum (ANP). Los métodos de prueba estándar para determinar las propiedades fisicoquímicas de las mezclas de biodiésel obtenidas de aceite de fritura y aceite de castor con combustible diésel permitieron establecer el cumplimiento de las especificaciones en Brasil, Europa y EE. UU. (Souza et al., 2011).

Al realizar investigaciones (Man et al., 2016) en un motor diésel (4 cilindros) de aspiración natural e inyección directa en un ciclo de prueba japonés de trece modos, utilizando combustible diésel puro, con mezclas de diésel puro con $10 \%, 20 \%$, $30 \%$ de biodiésel obtenido de aceite de fritura, y biodiésel puro, los resultados mostraron que las emisiones reguladas de hidrocarburos $(\mathrm{HC})$ y monóxido de carbono (CO) disminuyeron mientras que los óxidos de nitrógeno $\left(\mathrm{NO}_{\mathrm{x}}\right)$ se incrementaron para todas las condiciones de prueba cuando se compararon con el combustible diésel puro.

Un estudio experimental (Behcet et al., 2014) se realizó con mezclas de diésel puro con $25 \%$ de biodiésel producido de aceite usado de pescado y aceite de fritura usado. De acuerdo con los resultados, las mezclas de diésel puro con aceite usado de pescado mostraron los mejores parámetros de desempeño del motor y emisiones comparados con las mezclas de diésel puro con aceite de fritura usado. 


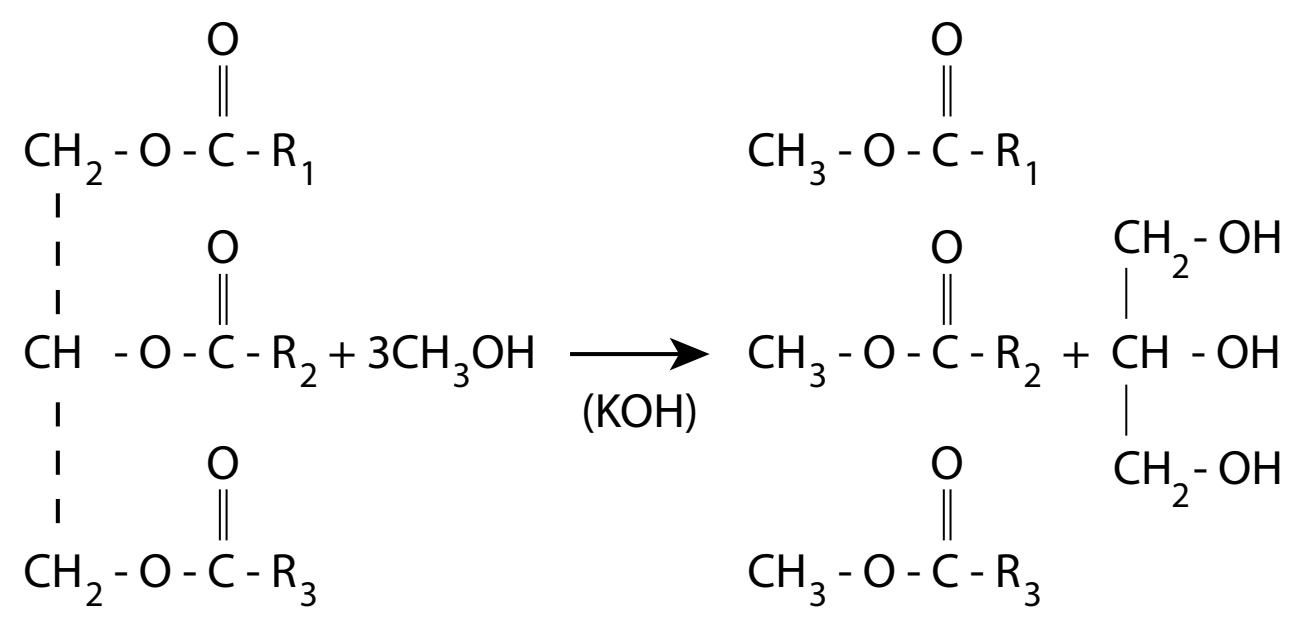

Figura 1. Transesterificacion del biodiésel

Fuente: Gopal et al. (2014)

Adaileh y AIQdah (2012) investigaron los efectos de uso de biodiésel obtenido de aceite de fritura usado sobre el desempeño de un motor diésel alimentado con biodiésel obtenido de aceite de fritura usado. Los resultados mostraron una reducción significativa de $\mathrm{CO}, \mathrm{HC}$ y las emisiones de humeado comparado con el combustible diésel estándar y B5.

C.S. Cheung et al. (2015) recurrieron a mezclas de diésel puro con biodiésel (B10, B20 y B30) obtenido de aceite de fritura usado, para estudiar su efecto en las emisiones. Según los resultados, el biodiésel permite reducir $\mathrm{HC}, \mathrm{CO}$ y la concentración de masa del material particulado, pero incrementa el $\mathrm{NO}_{x}$.

Los estudios sobre el uso de mezclas de diésel puro con biodiésel, el cual se obtuvo de aceite vegetal y de pescado usado, se realizaron en motores diésel. Estos funcionaron a velocidades variables (Man et al., 2016; Behcet et al., 2014; Adaileh y AlQdah, 2012; Corsini et al., 2016) y cargas variables correspondientes a la potencia efectiva y presión media efectiva a velocidad constante del motor (Man et al., 2016; Gopal et al., 2014; Cheung, 2015; Corsini et al., 2016).
En el presente estudio, el biodiésel fue producido con aceite de fritura usado con el objetivo de reutilizar aceite que normalmente es vertido por la población en los sistemas de desagüe y que contamina el agua y el medio ambiente.

Las mezclas de combustible diésel-biodiésel se utilizaron en un motor diésel para investigar sus efectos sobre el parámetro de emisión. Este último, al igual que el índice de opacidad, se midió durante el proceso de explotación en carga máxima a velocidades variables del motor entre 1000-1600 r.p.m. y cargas variables del motor correspondientes a la potencia efectiva a velocidad constante del motor a 1600 r.p.m. Los resultados de las pruebas experimentales fueron discutidos y comparados con los del combustible diésel.

\section{MATERIALES Y MÉTODOS}

Los experimentos se realizaron en un motor diésel de cuatro tiempos y un cilindro (figura 2). Las especificaciones del motor se detallan en la tabla 1. El motor provee una potencia máxima de 6,11 kW y está unido a un dinamómetro eléctrico de corriente continua, para controlar la velocidad de rotación y la carga aplicada. 


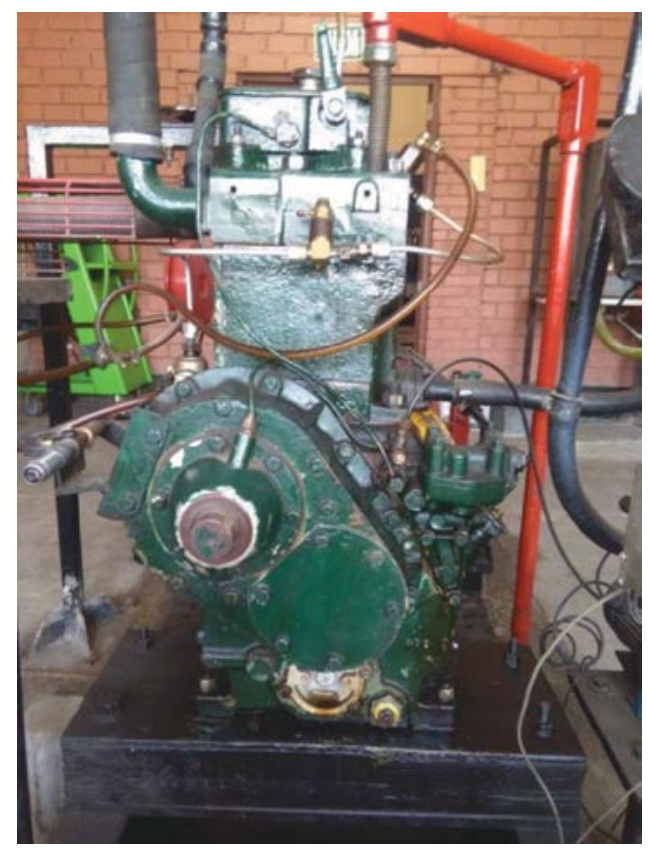

Figura 2. Motor diésel Petter

Fuente: elaboración propia

El combustible diésel puro se obtuvo directamente de la refinería local, mediante una solicitud para investigación, mientras que el biodiésel procedente de aceite de fritura usado se obtuvo de una planta de producción que funciona en una institución universitaria nacional, que lo procesa mediante la recolección de aceite de fritura usado de diferentes puntos de la ciudad de Lima.

En este estudio, el motor diésel utilizó combustible comercial B5 y mezclas de diésel puro con biodiésel de aceite de fritura usado al $10 \%$ (B10), $20 \%$ (B20) y $30 \%$ (B30) en volumen.

Un requerimiento importante para un combustible diésel es su fácil inflamación al entrar en contacto con el aire caliente; este se evalúa por el intervalo mínimo posible entre el instante en que se inyecta combustible y el instante en que se inflama, lo que se conoce como retardo a la inflamación. Por este motivo, cuanto más elevado es el índice de cetano, menor será el retardo a la inflamación.

Como método de reducción de humeado y toxicidad, se perfeccionan los procesos de formación de la mezcla y combustión de un motor (Lukanin, 1992) utilizando combustibles con elevado índice de cetano para disminuir el retardo de la inflamación y la rigidez del funcionamiento (Kumar et al., 2017). En la tabla 2 se presenta información obtenida en las pruebas de laboratorio, sobre las propiedades fisicoquímicas de los combustibles utilizados en las pruebas del motor. Se aprecia que los valores del índice de cetano para las mezclas B10, B20 y B30 son mayores en 4,6 \%, 3,9 \% y $4,6 \%$, respectivamente, a los valores asignados

Tabla 1. Datos técnicos del motor diésel

\begin{tabular}{ll}
\hline \multicolumn{1}{c}{ Marca } & \multicolumn{1}{c}{ Petter } \\
\hline Tipo de motor & Diésel de cuatro tiempos \\
\hline Modelo & PH1W, inyección directa \\
\hline Número de cilindros & Uno (1) \\
\hline Refrigeración & Por líquido \\
\hline Diámetro & $87,3 \mathrm{~mm}$ \\
\hline Carrera & $110 \mathrm{~mm}$ \\
\hline Cilindrada & $659 \mathrm{~cm}^{3}$ \\
\hline Relación de compresión & $16,5: 1$ \\
\hline Potencia máxima & $6,11 \mathrm{~kW}$ a 2000 r.p.m. \\
\hline Presión de inyección & $200 / 221 \mathrm{~kg} / \mathrm{cm}^{2}$ a $1100-2000$ r.p.m. \\
\hline Freno dinamométrico & Generador eléctrico de corriente continua \\
\hline
\end{tabular}

Fuente: elaboración propia. 
en la norma técnica vigente para el combustible comercial B5. La fuente principal del aceite y el proceso de cocción afectaron en la estructura molecular del aceite de fritura usado (Souza et al., 2011), la cual a su vez impactó en los valores elevados del índice de cetano mostrados en la tabla 2.
El contenido de oxígeno en el biodiésel es alto, por tanto, los valores de energía de masa son bajos comparados con el combustible diésel puro. Al aumentar la cadena de carbono del ácido graso (para un nivel de instauración constante), la fracción de masa de oxígeno disminuye y esto conlleva al

Tabla 2. Propiedades fisicoquímicas de los combustibles

\begin{tabular}{lccccccc}
\hline \multicolumn{1}{c}{ Propiedades } & Diésel & Diésel B5 & Diésel B5 (S-50) & B10 & B20 & B30 & B100 \\
\hline Índice de cetano & 50,8 & 49 & 46 & 51,4 & 51,0 & 51,4 & 44,0 \\
\hline Poder calorífico bruto, $\mathrm{MJ} / \mathrm{kg}$ & 45,213 & & & 48,014 & 45,141 & 45,486 & 41,396 \\
\hline Densidad, $\mathrm{kg} / \mathrm{l}$ & 0,8423 & & & 0,8459 & 0,8509 & 0,8556 & 0,8817 \\
\hline Viscosidad, $40^{\circ} \mathrm{C},(\mathrm{cSt})$ & 2,903 & $1,9-4,1$ & $1,9-4,1$ & 2,887 & 3,149 & 3,353 & 5,088 \\
\hline
\end{tabular}

Fuente: elaboración propia.

aumento del poder calorífico. En la tabla 2 se observa que el poder calorífico bruto de las mezclas B10 y B30 es mayor, comparado con el combustible diésel puro (Saxena et al., 2013).

Las pruebas experimentales se realizaron en condiciones estables en un motor diésel, de un cilindro, cuatro tiempos, con mezclas B10, B20 y B30, y el combustible diésel B5, en carga máxima para velocidades variables del motor entre 1000-1600 r.p.m. y cargas variables del motor correspondientes a la potencia efectiva a velocidad constante del motor de 1600 r.p.m.

El opacímetro Pierburg Instruments (figura 3) se utilizó para medir la opacidad del humeado. Después de que el motor alcanzó su temperatura de trabajo, la sonda del opacímetro fue instalada en el tubo de escape. Las especificaciones técnicas del opacímetro se presentan en la tabla 3.

\section{RESULTADOS}

El estudio experimental busca investigar el efecto del combustible obtenido de aceite de fritura usado, sobre el índice de opacidad, lo cual se realizó en un motor diésel. Para llevar a cabo el experimento, se emplearon cuatro combustibles: B5, B10, B20 y B30 en un motor de prueba.

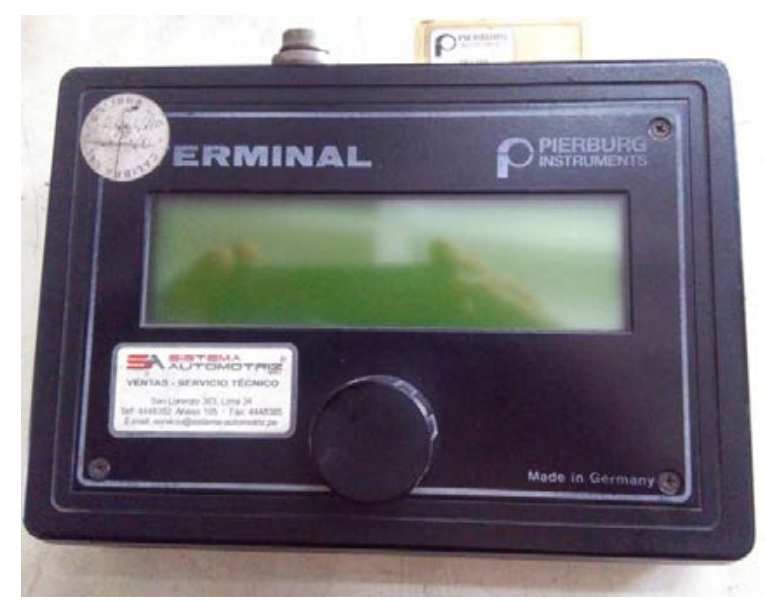

Figura 3. Opacímetro Pierburg Instruments

Fuente: elaboración propia.

Tabla 3. Especificaciones del opacímetro

\begin{tabular}{ll}
\hline Marca & Pierburg Instruments \\
\hline Modelo & DO -285 \\
\hline Procedencia & Alemania \\
\hline Serie & 6022 \\
\hline Tensión & $230 \mathrm{~V}$ \\
\hline Frecuencia & $50-60 \mathrm{~Hz}$ \\
\hline Rango de opacidad & de 0 a $9,99 \mathrm{~m}^{-1}$ \\
\hline Rango de temperatura & de 0 a $120^{\circ} \mathrm{C}$ \\
\hline Velocidad del motor & 400 a 6000 r.p.m. \\
\hline
\end{tabular}

Fuente: elaboración propia. 
Las características de los combustibles, la ratio de atomización, la forma de la cámara de combustión, la frecuencia de rotación del motor, la relación aire/combustible y otros parámetros operativos afectan en la cantidad de las emisiones de los motores (Behcet et al., 2014).

La variación del índice de opacidad en función de la frecuencia de rotación del motor de los combustibles utilizados se muestra en la figura 4 . El índice de opacidad es menor a bajas frecuencias de rotación (1000-1200 r.p.m.), al incrementar el contenido de biodiésel en el combustible diésel puro. Esto se puede atribuir al mayor índice de cetano y contenido de oxígeno en el biodiésel (Gopal et al., 2014; Cheung et al., 2015; Man et al., 2016). Sin embargo, a frecuencias de rotación media (1200-1400 r.p.m.), el índice de opacidad de la mezcla B10 se incrementa en mayor medida, en comparación con las otras tres mezclas. Esta tendencia de B10 también a frecuencia de rotación alta (1400-1600 r.p.m.), podría atribuirse al bajo contenido de oxígeno adicional en la mezcla y al menor tiempo de duración del proceso de combustión al incrementarse la frecuencia de rotación del motor. El índice de opacidad de los combustibles B10, B20 y B30 es menor en 0,3 \%, 24 \% y $46 \%$, respectivamente, comparado con el combustible comercial B5 cuando el motor alcanza la frecuencia de rotación correspondiente a 1600 r.p.m.

Behcet et al. (2014) mencionan que, al incrementar la frecuencia de rotación del motor, aumenta el chorro de aire, los movimientos rotacionales y de turbulencia, los cuales optimizan homogeneidad a la carga y provocan una combustión más completa; así mismo, se reporta la disminución de la opacidad al incrementarse la frecuencia de rotación del motor en las pruebas realizadas al recurrir, como combustibles, al biodiésel de aceite de pescado y al biodiésel de aceite usado.

Según la figura 4, la opacidad se incrementa al aumentar la frecuencia de rotación para todos los combustibles utilizados, lo que podría atribuirse al contenido de componentes aromáticos en estos, y a la calidad de atomizado del dardo de combustible.

En la figura 5, se muestra la variación del índice de opacidad y la potencia efectiva de las mezclas diésel/biodiesel, comparadas con el combustible diésel comercial B5 para diferentes cantidades de toma de datos experimentales a frecuencia de rotación constante del motor de 1600 r.p.m. La tendencia del índice de opacidad es similar para B5, B10, B20 y B30, a bajas cargas a pesar del incremento de

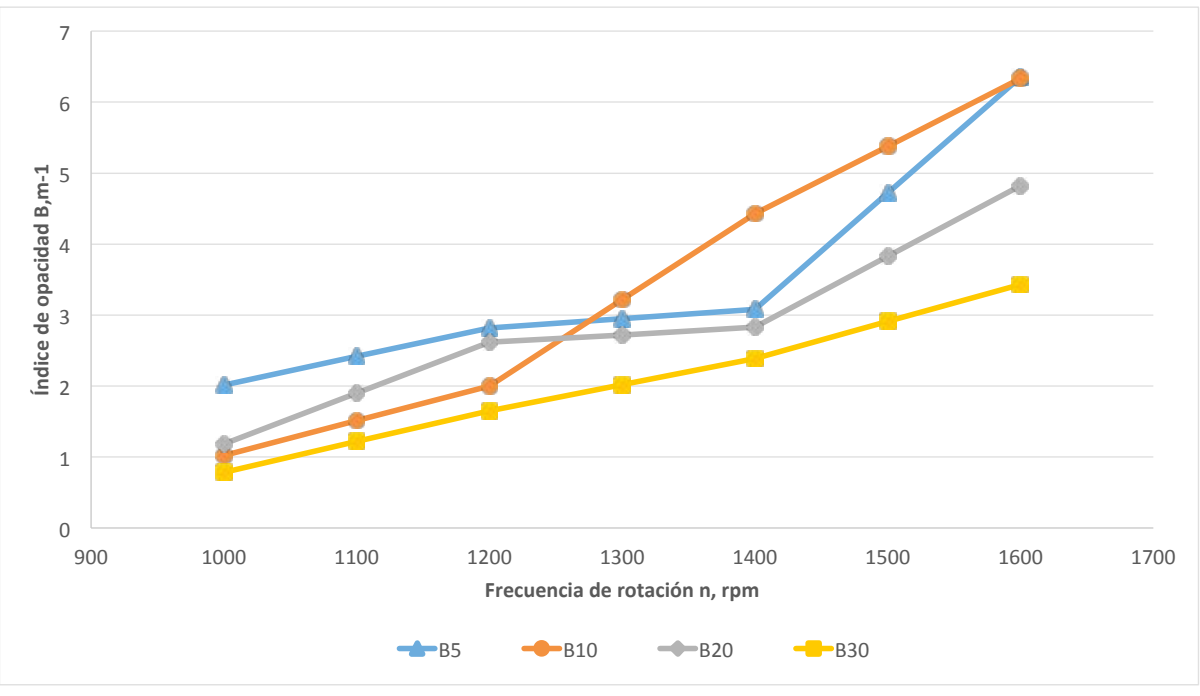

Figura 4. Variación de la opacidad en función de la frecuencia de rotación

Fuente: elaboración propia. 
la viscosidad (Corsini et al., 2016) de las mezclas con mayor contenido de biodiésel comparado con el B5, pero a carga media el máximo valor corresponde a B5. Por otro lado, a cargas altas del motor, el mayor valor de índice de opacidad corresponde a B20 en comparación con las otras mezclas. Las tendencias de variación del índice de opacidad de todos los combustibles utilizados según la figura 5, son afectados por la baja relación de carbono/hidrógeno, contenido de oxígeno en las mezclas diésel/biodiésel y la carga sobre el motor (Gopal et al., 2014; Cheung et al., 2015).

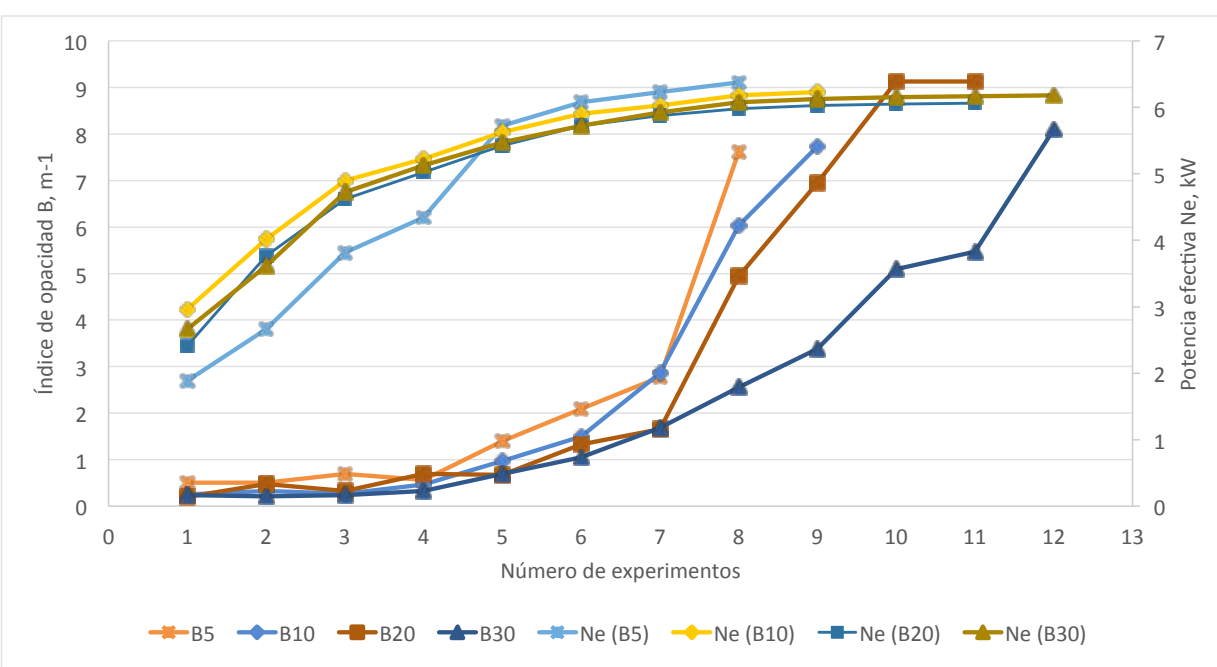

Figura 5. Variación del índice de opacidad y potencia efectiva en función de número de experimentos

Fuente: elaboración propia.

\section{CONCLUSIONES}

La investigación se basa en la influencia del biodiésel obtenido de aceite de fritura usado, sobre el índice de opacidad. Las pruebas se realizaron a diferentes condiciones de operación del motor diésel de prueba.

Las mediciones de opacidad obtenidas con las mezclas B10, B20 y B30 en el régimen nominal de revoluciones del motor de 1600 r.p.m. a carga constante, son menores comparados con el combustible comercial B5 en 0,3\%, $24 \%$ y $46 \%$, respectivamente.

Al incrementar la carga del motor a velocidad constante de 1600 r.p.m., el índice de opacidad alcanza su máximo valor correspondiente a la mezcla B20, comparado con los otros combustibles utilizados.
Como resultado, es viable el uso de biodiésel de aceite de fritura usado, en mezclas con diésel puro y comercial para disminuir las emisiones tóxicas como el material particulado (hollín) de los motores diésel en el medio ambiente. Por tanto, la reutilización del aceite de fritura disminuirá significativamente los problemas medioambientales.

\section{AGRADECIMIENTOS}

Los autores agradecen al Instituto de Motores de Combustión Interna de la Facultad de Ingeniería Mecánica de la Universidad Nacional de Ingeniería, por las facilidades otorgadas para la realización y procesamiento de las pruebas. 


\section{REFERENCIAS}

Adaileh, W. y AlQdah, K. (2012). Performance of Diesel Engine Fuelled by a Biodiesel Extracted from a Waste Cocking Oil. Energy Procedia, 18, 1317- 1334. DOI: https://doi.org/10.1016/j. egypro.2012.05.149

Arango, J.E. et al. (2014). Análisis exploratorio de investigaciones sobre los motores de combustión interna que trabajan con biogás. Tecnura, 18, 152164. DOI: https://doi.org/10.14483/udistrital.jour. tecnura.2014.1.a11

Behcet, R. et al. (2014). Effects of Fuels Produced from Fish and Cooking Oils on Performance and Emissions of a Diesel Engine. Energy, 71, 645-655. DOI: https://doi.org/10.1016/j.energy.2014.05.003

Canesin, E. et al. (2014). Characterization of Residual Oils for Biodiesel Production. Electronic Journal of Biotechnology, 17, 39-45.

Cheung, C. et al. (2015). Effect of Waste Cooking Oil Biodiesel on the Emissions of a Diesel Engine. Energy Procedia, 66, 93-96. DOI: https://doi.org/10.1016/j.egypro.2015.02.050

Corsini, A. et al. (2016). Performance Analysis of a Common-Rail Diesel Engine Fuelled with Different Blends of Waste Cooking Oil and Gasoil. Energy Procedia, 101, 606-613. DOI: https://doi. org/10.1016/j.egypro.2016.11.077

Gopal, K.N. et al. (2014). Investigation of Emissions and Combustion Characteristics of a $\mathrm{Cl}$ Engine Fueled with Waste Cooking Oil Methyl Ester and Diesel Blends. Alexandria Engineering Journal, 53, 281- 287. DOI: https://doi.org/10.1016/j. aej.2014.02.003

Kumar, M. et al. (2017). The Impacts on Combustion, Performance and Emissions of Biodiesel by Using Additives in Direct Injection Diesel Engine. Alexandria Engineering Journal, 57(1). DOI: https://doi.org/10.1016/j.aej.2016.12.016

Lukanin, V. (1992). Motores de combustión interna. Moscú, Rusia: Editorial MIR.

Man, X.J. et al. (2016). Influence of Engine Load and Speed on Regulated and Unregulated Emissions of a Diesel Engine Fueled with Diesel Fuel Blended with Waste Cooking Oil Biodiesel. Fuel, 180, 41-49. DOI: https://doi.org/10.1016/j.fuel.2016.04.007

Nabanita, B. et al. (2014). Biodiesel Production from Used Vegetable Oil from Shops Selling Fritters in Kolkata. Energy Procedia, 54, 161-165. DOI: https://doi.org/10.1016/j.egypro.2014.07.259

Núñez, D.W. (2012). Uso de residuos agrícolas para la producción de biocombustibles en el departamento del Meta. Tecnura, 16, 142-156. DOI: https://doi. org/10.14483/udistrital.jour.tecnura.2012.4.a10

Saxena, P. et al. (2013). A review on prediction of properties of biodiesel and blends of biodiesel. Procedia Engineering, 51, 395-402. DOI: https://doi. org/10.1016/j.proeng.2013.01.055

Souza, O. et al. (2011). Physical- Chemical properties of Waste Cooking Oil Biodiesel and Castor Oil Biodiesel Blends. Fuel, 90, 1700-1702. DOI: https:// doi.org/10.1016/j.fuel.2010.10.045 